\title{
Impact of QTL minor allele frequency on genomic evaluation using real genotype data and simulated phenotypes in Japanese Black cattle
}

Yoshinobu Uemoto $^{1 *}$, Shinji Sasaki ${ }^{1}$, Takatoshi Kojima', Yoshikazu Sugimoto ${ }^{2}$ and Toshio Watanabe ${ }^{1}$

\begin{abstract}
Background: Genetic variance that is not captured by single nucleotide polymorphisms (SNPS) is due to imperfect linkage disequilibrium (LD) between SNPs and quantitative trait loci (QTLS), and the extent of LD between SNPs and QTLs depends on different minor allele frequencies (MAF) between them. To evaluate the impact of MAF of QTLS on genomic evaluation, we performed a simulation study using real cattle genotype data.
\end{abstract}

Methods: In total, 1368 Japanese Black cattle and 592,034 SNPs (Illumina BovineHD BeadChip) were used. We simulated phenotypes using real genotypes under different scenarios, varying the MAF categories, QTL heritability, number of QTLs, and distribution of QTL effect. After generating true breeding values and phenotypes, QTL heritability was estimated and the prediction accuracy of genomic estimated breeding value (GEBV) was assessed under different SNP densities, prediction models, and population size by a reference-test validation design.

Results: The extent of LD between SNPs and QTLs in this population was higher in the QTLs with high MAF than in those with low MAF. The effect of MAF of QTLs depended on the genetic architecture, evaluation strategy, and population size in genomic evaluation. In genetic architecture, genomic evaluation was affected by the MAF of QTLs combined with the QTL heritability and the distribution of QTL effect. The number of QTL was not affected on genomic evaluation if the number of QTL was more than 50. In the evaluation strategy, we showed that different SNP densities and prediction models affect the heritability estimation and genomic prediction and that this depends on the MAF of QTLs. In addition, accurate QTL heritability and GEBV were obtained using denser SNP information and the prediction model accounted for the SNPs with low and high MAFs. In population size, a large sample size is needed to increase the accuracy of GEBV.

Conclusion: The MAF of QTL had an impact on heritability estimation and prediction accuracy. Most genetic variance can be captured using denser SNPs and the prediction model accounted for MAF, but a large sample size is needed to increase the accuracy of GEBV under all QTL MAF categories.

Keywords: BovineHD, Genomic prediction, Heritability estimation, Japanese Black cattle, Minor allele frequency, Simulation study

\footnotetext{
*Correspondence: y0uemoto@nlbc.go.jp

'National Livestock Breeding Center, Nishigo, Fukushima 961-8511, Japan

Full list of author information is available at the end of the article
} 


\section{Background}

The development of single nucleotide polymorphism (SNP) array technology has enhanced the genetic dissection of complex traits, and this SNP information can be directly utilized in cattle breeding programs using genomic selection $[1,2]$. In addition, whole genome sequence (WGS) data are becoming increasingly available for cattle, and WGS data are expected to yield a better understanding of complex traits, which can capture all of the genetic variance and predict an accurate genomic estimated breeding value (GEBV), by accounting for all the variants including quantitative trait loci (QTLs) [3, 4].

A recent report showed that the SNPs significantly associated with a complex trait explain only a fraction of the phenotypic variance in human height, and this has been called the "missing heritability" problem [5]. It has been argued that missing heritability is due to imperfect linkage disequilibrium (LD) between SNPs and QTLs, and the extent of LD between SNPs and QTLs depends on differences in the minor allele frequency (MAF) between SNPs and QTLs [6]. SNPs with similar MAF can potentially have high LD, but SNPs with very different MAF cannot have high LD. In cattle populations, QTLs may have a lower MAF than SNPs on low-density SNP arrays, because these are designed to work in several different breeds. In this case, the genetic variation explained by SNPs will be lower than that due to low LD between SNPs and QTLs with low MAF. Meat from Japanese Black cattle is known to have the unique characteristic of a high degree of marbling; the cattle are genetically distant from other European breeds at the genome level [7]. The extent of LD between SNPs and QTLs in Japanese Black cattle may differ from that in other cattle breeds, and it is necessary to evaluate the impact of MAF of QTLs on the genomic evaluation in this target population.

Heritability estimation and GEBV prediction are measures of goodness-of-fit in reference populations and have predictive ability in test populations, respectively. The amount of genetic variance not captured by SNPs affects the maximum predictive ability [8]. On the other hand, increasing the goodness-of-fit will not necessarily increase the predictive ability, because of the model over-fitting problem [9]. The heritability estimation and prediction accuracy depend on several factors such as the genetic architecture of a trait (e.g., QTL heritability, number of QTLs, and distribution of QTL effect), the evaluation strategy (e.g., SNP marker density and prediction method), and population size [6,9-12]. Therefore, it is important how heritability estimation and GEBV prediction depends on these factors in different MAF of QTLs.

The objective of this study was to evaluate the impact of MAF of QTLs on heritability estimation and accuracy of GEBV prediction, and how that depends on the genetic architecture (QTL heritability, number of QTLs, and distribution of QTL effect), the evaluation strategy (SNP density and prediction model), and population size. We performed a simulation analysis based on a referencetest validation design, which used real genotype data to account for the extent of LD in Japanese Black cattle.

\section{Methods}

Genotypes for this study were obtained from previously published data [13]. All animal experiments were performed according to the Guidelines for the Care and Use of Laboratory Animals of Shirakawa Institute of Animal Genetics, and this research was approved by Shirakawa Institute of Animal Genetics Committee on Animal Research (H21-2). We have obtained the written agreement from the cattle owners to use the samples.

\section{Data}

In this simulation analysis, real genotype data were used to account for the extent of LD in Japanese Black cattle. Complete descriptions of the experimental population and SNP information were reported previously by Uemoto et al. [13]. Briefly, a total of 1444 Japanese Black cattle, which were 653 steers from two slaughterhouses in Japan [14] and 791 cows from farms managed by a large cooperative farming company in Japan [15], were genotyped using the Illumina BovineHD BeadChip (HD) (Illumina, San Diego, CA, USA), and 593,696 SNPs on autosomal chromosomes assessed by the exclusion criteria of $\mathrm{MAF}<0.01$, call rate $<0.95$, and Hardy-Weinberg equilibrium test $<0.001$ were used in this study. To avoid having very close relatives in the data, the animals with large off-diagonal elements in the genomic relationship matrix (GRM) were excluded (a cut-off value of \pm 0.4 for offdiagonal elements), and the SNPs were then reassessed by the same criteria. A total of 1368 animals and 592,034 SNPs were then used in the simulation study. These animals were low relatives with the progeny of 438 sires, and the mean, median, and maximum number of progenies per sire were $3.1,2$, and 24, respectively. The distribution of progenies per sire was shown in Additional file 1: Figure S1.

\section{Simulation design}

In this study, we simulated the true breeding value (TBV) and phenotypes under the different scenarios varying the following factors: different MAF categories, QTL heritability, number of QTLs, and distribution of QTL effect. After generating TBV and phenotypes, the QTL heritability was estimated and the prediction accuracy of GEBV was assessed under different conditions varying the following factors: different SNP densities, prediction models, and size of the reference-test populations by a reference-test validation design. The factors considered in the simulation study are summarized in 
Table 1, and shown in detail below. The impact of the MAF of QTLs on genomic evaluation under different genetic architecture was evaluated in scenarios 1 and 2 . In addition, the impact of the MAF of QTLs on genomic evaluation under different evaluation strategy and population size was evaluated in scenarios 3 and 4, respectively.

In this simulation, 36,478 and 6316 SNPs on the BovineSNP50v2 BeadChip (50 K) and the BovineLDv1.1 BeadChip (7 K) (Illumina, San Diego, CA, USA), respectively, were designated as SNP markers. The distribution density of MAF of SNPs on $7 \mathrm{~K}, 50 \mathrm{~K}$, and HD is plotted in Fig. 1. The MAF distribution shows a low ratio of SNPs on $7 \mathrm{~K}$ and a high ratio of SNPs on $50 \mathrm{~K}$ and HD at low MAF. The remaining 555,556 SNPs that are present in the $\mathrm{HD}$ but not in the $50 \mathrm{~K}$ and $7 \mathrm{~K}$ were assumed as candidate QTLs. For SNP density, three types of SNPs were used in this simulation. First, SNPs on $7 \mathrm{~K}$ and $50 \mathrm{~K}$ were used, and this scenario involved imperfect LD between SNPs and QTLs (and named as the imperfect LD SNPs). Second, the HD genotype was imputed from SNPs on $50 \mathrm{~K}$ (50 K_to_HD) and $7 \mathrm{~K}$ (7K_to_HD) by the BEAGLE (v4.0) software [16]. We performed a 10-fold crossvalidation to have imputed HD genotype in this population, and the detail of imputation was reported previously by Uemoto et al. [13]. The imputed SNPs were then reassessed by the same exclusion criteria as described above, and 585,015 and 588,547 SNPs were used in the 7K_to_HD and 50 K_to_HD, respectively. The detail of the imputation error ratio was shown by Uemoto et al. [13], and the average correlation between true and imputed genotypes were 0.98 in 50 K_to_HD and 0.93 in 7 K_to_HD. This scenario involved some SNPs being QTLs but with a low imputation error ratio (and named as the imputed SNPs). Third, all SNPs on the HD were used as SNPs, and this scenario assumed that WGS data were available and some SNPs were QTLs itself (and named as the perfect LD SNPs).
For candidate QTLs, three MAF categories were defined as follows: a low MAF group $(0.01 \leq \mathrm{MAF} \leq 0.05)$, a high MAF group $(0.05<$ MAF $\leq 0.5)$, and an all MAF group $(0.01 \leq \mathrm{MAF} \leq 0.5)$. A total of $50,100,300,500$, 1000 , and 2000 QTLs were randomly selected from candidate QTLs in each MAF group. Hill et al. [17] showed that the distribution of allele frequency affecting additive genetic variance is under the U-shaped distribution and $f(p) \propto \frac{1}{p(1-p)}$. For the all MAF group, the U-shaped distribution was assumed as the distribution of QTL allele frequency $(0.01 \leq p \leq 0.5)$, and the ratio of the integrated values for low MAF, $\int_{0.01}^{0.05} f(p) d p$, and high MAF, $\int_{0.05}^{0.5} f(p) d p$, were 0.36 and 0.64 , respectively. Therefore, QTLs with low and high MAFs in the all MAF group were randomly selected from the ratio 0.36:0.64, respectively.

We assumed the use of a polygenic model in the simulation, because this is a reasonable assumption for the majority of complex traits in cattle. The phenotype was simulated by summing all true QTL genotypic values and the residual effect, that is, $y_{i}=\sum_{j}^{m} x_{i j} b_{j}+e_{i}$, where $m$ is the number of QTLs, $x_{i j}$ is the genotype for the $j$-th QTL of the $i$-th animal (coded as 0,1 , or 2 for the homozygote, heterozygote, and the other homozygote, respectively), $b_{j}$ is the allele substitution effect of the $j$-th QTL, and $e_{i}$ is the residual effect generated from $N\left(0, \sigma_{g}^{2}\left(1 / h^{2}-1\right)\right) \cdot \sum_{j}^{m} x_{i j} b_{j}$ is TBV, $\sigma_{g}^{2}$ is the total genetic variance of TBV, and $h^{2}$ is the setting value of QTL heritability. Three setting values of QTL heritability $\left(h^{2}=\right.$ $0.20,0.40$, and 0.80 ) were used to generate phenotypes.

In this study, two different distributions of the QTL effect were assumed. The first model was a gamma distribution

Table 1 Factors for different scenarios in a simulation study

\begin{tabular}{|c|c|c|c|c|}
\hline \multirow[b]{2}{*}{ Factor } & \multicolumn{4}{|l|}{ Scenario } \\
\hline & 1 & 2 & 3 & 4 \\
\hline$\overline{M^{\prime} F^{a}}$ & All, High, Low & All, High, Low & All, High, Low & All, High, Low \\
\hline QTL heritability & $0.2,0.4,0.8$ & 0.4 & 0.4 & 0.4 \\
\hline Number of QTLs & 500 & $50,100,300,500,1000,2000$ & 500 & 500 \\
\hline Distribution of QTL effect ${ }^{\mathrm{b}}$ & EquV & Gamma, EquV & EquV & EquV \\
\hline SNP density ${ }^{c}$ & $50 \mathrm{~K}$ & $50 \mathrm{~K}$ & $7 \mathrm{~K}, 50 \mathrm{~K}, 7 \mathrm{~K}$ _to_HD, $50 \mathrm{~K} \_$to_HD, HD & $50 \mathrm{~K}$ \\
\hline Prediction model $^{d}$ & Model (1) with $G_{Y}$ & Model (1) with $G_{Y}$ & Model (1) with $G_{V}, G_{Y}$, and $G_{S}$, Model (2) & Model (1) with $G_{Y}$ \\
\hline Size of reference set & 1231 & 1231 & 1231 & $200,400,800,1200$ \\
\hline Size of test set & 137 & 137 & 137 & $1168,968,568,168$ \\
\hline
\end{tabular}

${ }^{a} \mathrm{MAF}$, Minor allele frequency; All, $0.01 \leq \mathrm{MAF} \leq 0.5$; High, $0.05<\mathrm{MAF} \leq 0.5$; Low, $0.01 \leq \mathrm{MAF} \leq 0.05$

${ }^{\mathrm{b}}$ Gamma, Gamma distribution model; EquV, Equal variance model

${ }^{\mathrm{C}} 7 \mathrm{~K}, 50 \mathrm{~K}$ and HD, Illumina infinium BovineLDv1.1, BovineSNP50v2, and BovineHD BeadChips, respectively; 7 K_to_HD and 50 K_to_HD, Imputations were performed from $7 \mathrm{~K}$ and $50 \mathrm{~K}$ to $\mathrm{HD}$, respectively

${ }^{d} G_{V}$, VanRaden's $G$ matrix; $G_{Y}$, Yang's $G$ matrix; $G_{\mathrm{s}}$, Speed's $G$ matrix 


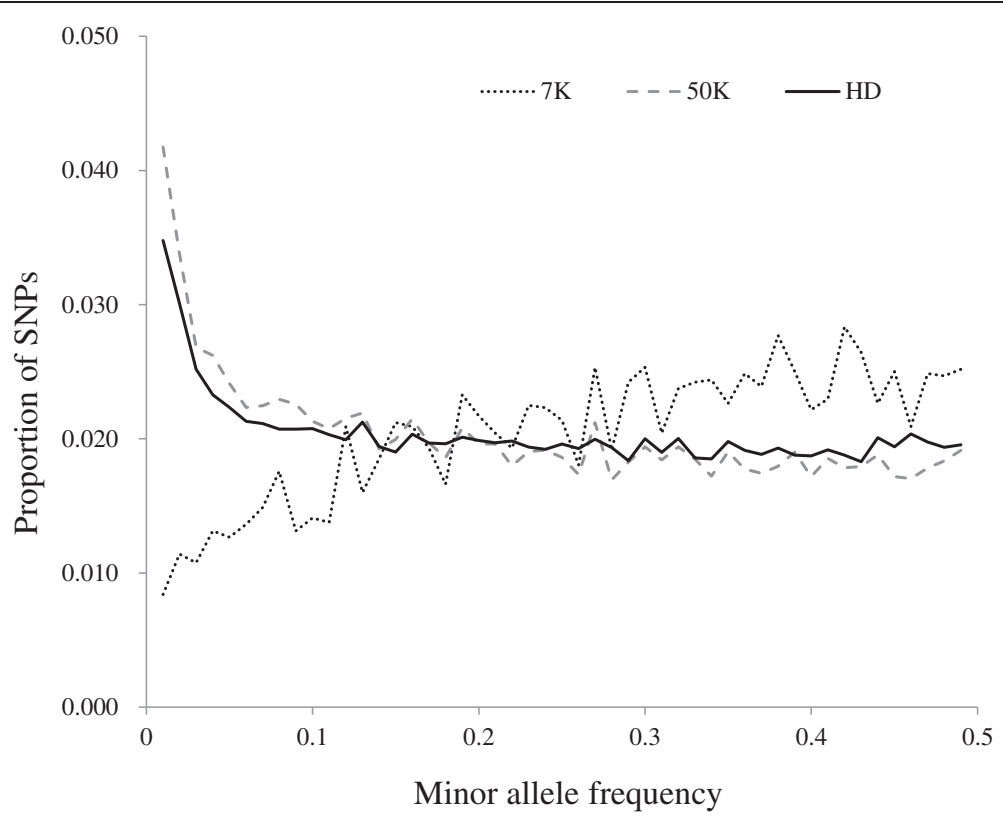

Fig. 1 Distribution of minor allele frequencies for SNPs under different SNP densities. The $x$-axis indicates the MAF of SNPs, and the $y$-axis represents the proportion of SNPs in each MAF category. 7 K, 50 K, and HD are SNP markers on Illumina infinium BovineLDv1.1, BovineSNP50v2, and BovineHD BeadChips, respectively

model in which the QTL effect was generated from a gamma distribution with a shape parameter of 0.4 and scale parameter of 1.66 [2]. The second model was an equal variance model in which the QTL effect was assumed as $b_{j}=\frac{1}{\sqrt{2 p_{j}\left(1-p_{j}\right)}}$, where $p_{j}$ is MAF of $j$-th QTL. In the equal variance model, the QTL effect was assumed in that all QTLs had contributed to QTL variance equally $\left(\operatorname{Var}\left(b_{j}\right)=1\right.$ in this assumption) if linkage equilibrium was assumed among QTLs. The signs of QTL effects were randomly selected, and total QTL variance was adjusted to $100 \times h^{2}$ in both distribution models.

\section{Statistical analysis}

The generated data were analyzed by the genomic best linear unbiased prediction (GBLUP) method with the following model:

$$
\mathbf{y}=\mathbf{1}_{\mathbf{n}} \mu+\mathbf{X} \mathbf{u}+\mathbf{e}
$$

where $\mathbf{y}$ is the phenotypic values, $\mathbf{1}_{\mathbf{n}}$ is a vector of $n$ ones, $\mu$ is the mean, $\mathbf{X}$ is the design matrix for random effects, $\mathbf{u}$ is the additive genetic effect with $\mathbf{u} \sim N$ $\left(0, \mathbf{G} \sigma_{u}^{2}\right)$, and $\mathbf{e}$ is the residual effect with $\mathbf{e} \sim N\left(0, \mathbf{I} \sigma_{e}^{2}\right)$. $\mathbf{G}$ is a GRM using all SNPs in each SNP density. ${ }_{u}^{2}$ is the additive genetic variance, and $\sigma_{e}^{2}$ is residual variance. We also used the following model:

$$
\mathbf{y}=\mathbf{1}_{\mathbf{n}} \mu+\mathbf{X} \mathbf{u}_{\mathbf{L}}+\mathbf{X} \mathbf{u}_{\mathbf{H}}+\mathbf{e}
$$

where $\mathbf{u}_{\mathbf{L}}$ is the additive genetic effect attributed to the low MAF SNPs with $\mathbf{u}_{\mathbf{L}} \sim N\left(0, \mathbf{G}_{\mathbf{L}} \sigma_{u_{L}}^{2}\right)$, and $\mathbf{u}_{\mathbf{H}}$ is the additive genetic effect attributed to the high MAF SNPs with $\mathbf{u}_{\mathbf{H}} \sim N\left(0, \mathbf{G}_{\mathbf{H}} \sigma_{u_{H}}^{2}\right) . \mathbf{G}_{\mathbf{L}}$ is a GRM using SNPs with low MAF, and $\mathbf{G}_{\mathbf{H}}$ is a GRM using SNPs with high MAF in each SNP density. $\sigma_{u_{L}}^{2}$ and $\sigma_{u_{H}}^{2}$ are the additive genetic variances attributed to the SNPs with low and high MAFs, respectively, and $\sigma_{e}^{2}$ is the residual variance. We defined three different GRMs as follows:

VanRaden's GRM $\left(\mathbf{G}_{\mathbf{V}}\right)$ : The first GRM, $\mathbf{G}_{\mathbf{V}}$, was proposed by VanRaden [18] and is calculated as follows:

$$
\mathbf{G}_{\mathbf{v}}=\frac{\mathbf{Z} \mathbf{Z}^{\prime}}{2 \sum_{j=1}^{m} p_{j}\left(1-p_{j}\right)}
$$

where $m$ is the number of SNPs, $p_{j}$ is the frequency of the second allele of $j$-th SNP, and the elements of $Z$ are calculated as follows:

$$
z_{i j}=x_{i j}-2 p_{j}
$$

where $x_{i j}$ is the number of the second allele of the $i$-th individual at the $j$-th SNP.

Yang's GRM $\left(\mathbf{G}_{\mathbf{Y}}\right)$ : The second GRM, $\mathbf{G}_{\mathbf{Y}}$, was proposed by Yang et al. [6] and is computed as follows:

$$
\mathbf{G}_{\mathbf{Y}}=\frac{\overline{\mathbf{Z}} \overline{\mathbf{Z}}^{\prime}}{m}
$$

where $\overline{\mathbf{Z}}$ is the $\mathbf{Z}$ matrix but with each element scaled based on the allele frequency of each locus as follows: 


$$
\bar{z}_{i j}=\frac{z_{i j}}{\sqrt{2 p_{j}\left(1-p_{j}\right)}}
$$

Speed's GRM $\left(\mathbf{G}_{\mathbf{S}}\right)$ : The third GRM, $\mathbf{G}_{\mathbf{S}}$, was proposed by Speed et al. [19] and is calculated as follows:

$$
\mathbf{G}_{S}=\frac{\mathbf{W} \mathbf{W}^{\prime}}{\sum_{j=1}^{m} k_{j}}
$$

where $k_{j}$ is the weighting factor of the $j$-th SNP accounted for LD and the elements of $\mathbf{W}$ are calculated as follows:

$$
w_{i j}=\sqrt{k_{j}} \bar{z}_{i j}
$$

Speed et al. [19] proposed a method for weighting markers to account for LD. Their method, linkagedisequilibrium adjusted kinships (LDAK), examines the local SNP correlation caused by LD and computes optimal SNP weights by solving a linear program. We calculated the weighting factor $k_{j}$ and the LD-adjusted GRM $\left(\mathbf{G}_{\mathbf{S}}\right)$ by the LDAK software with default parameters and LD decay function. When analyzing high density SNPs (i.e., imputed SNPs and perfect LD SNPs), the weighting factors were calculated twice as suggested.

After calculating these three GRMs, 0.00001 was added to diagonal elements of each GRM to avoid near singularity problems. We used the three GRMs in model (1) and $\mathbf{G}_{\mathbf{Y}}$ in model (2). The QTL heritability $h_{1}^{2}$ and $h_{2}^{2}$ for model (1) and (2), respectively, are calculated as follows,

$$
\begin{aligned}
& h_{1}^{2}=\frac{\sigma_{u}^{2}}{\sigma_{u}^{2}+\sigma_{e}^{2}} \\
& h_{2}^{2}=\frac{\sigma_{u_{L}}^{2}+\sigma_{u_{H}}^{2}}{\sigma_{u_{L}}^{2}+\sigma_{u_{H}}^{2}+\sigma_{e}^{2}}
\end{aligned}
$$

\section{Validation test of heritability estimation and prediction accuracy}

Under each scenario, we replicated a reference-test validation design 300 times. In each reference-test experiment, data were randomly split into two disjointed sets, that is, 137 animals (one-tenth of all animals) in the test population and the remaining 1231 animals in the reference population. In each replica, this approach was performed only one time. In addition, to evaluate the impact of MAF of QTLs under different population size, $200,400,800$, and 1200 animals were randomly selected as the reference population, and the remaining 1168 , 968,568 , and 168 animals were used as the test population, respectively. Phenotypes of animals in the test population were masked in each replicate, and we estimated QTL heritability in the reference population and predicted the GEBV in the test population using the ASREML 3.0 program [20]. After predicting the GEBV, the prediction accuracy was assessed using Pearson's correlation between TBV and GEBV in each test population of the validation set. The mean and standard deviation (SD) of 300 replicates was then calculated.

\section{Results}

\section{Extent of LD between SNPs and QTLs}

Under all scenarios, three MAF categories were defined to evaluate the impact of MAF of QTLs. To evaluate the impact of MAF of QTLs on the extent of LD between SNPs and QTLs, the extent of LD between SNPs on $50 \mathrm{~K}$ and QTLs in each MAF category is shown in Fig. 2. The extent of LD between SNPs and QTLs was evaluated using the $r^{2}$ value, which is a measure of LD. The $r^{2}$ values between QTLs and both adjacent SNPs were calculated by PLINK software [21]. The maximum value of $\mathrm{r}^{2}$ between two QTL-SNP intervals was chosen in each QTL, and the density distributions of $\mathrm{r}^{2}$ for three MAF categories were then plotted. The parameters used were the same as those used in scenario 1. In this result, most QTLs with low MAF had a lower $r^{2}$ value than those with high MAF. The $r^{2}$ value of QTLs with all MAF was between that of QTLs with low and high MAFs. The mean values of $\mathrm{r}^{2}$ for all, high, and low MAFs were $0.294,0.360$, and 0.184 , respectively. This shows that the extent of LD between SNPs and QTLs is higher in the QTLs with high MAF than that in those with low MAF.

\section{The genetic architecture}

We evaluated the impact of MAF of QTLs on genomic evaluation under different QTL heritability in scenario 1, and the estimated QTL heritability and correlation between TBV and GEBV are shown in Fig. 3. The estimated QTL heritability was close to the setting value and a higher correlation was observed as the QTL heritability was increased in each MAF category. For the MAF of QTLs, the estimated QTL heritability and correlation between TBV and GEBV for QTLs with high MAF has the highest value, and the values of all MAF were between those of low and high MAFs in each setting value of QTL heritability. In addition, as the setting value was increased from 0.20 to 0.80 , the differences in the results between high and low MAFs increased in QTL heritability (from 0.06 to 0.15 , respectively) and correlation between TBV and GEBV (from 0.14 to 0.16 , respectively).

We evaluated the impact of MAF of QTLs on genomic evaluation under different number of QTLs and distribution of the QTL effect in scenario 2, and the estimated QTL heritability and correlation between TBV and GEBV are shown in Fig. 4. For QTL number, the estimated QTL 


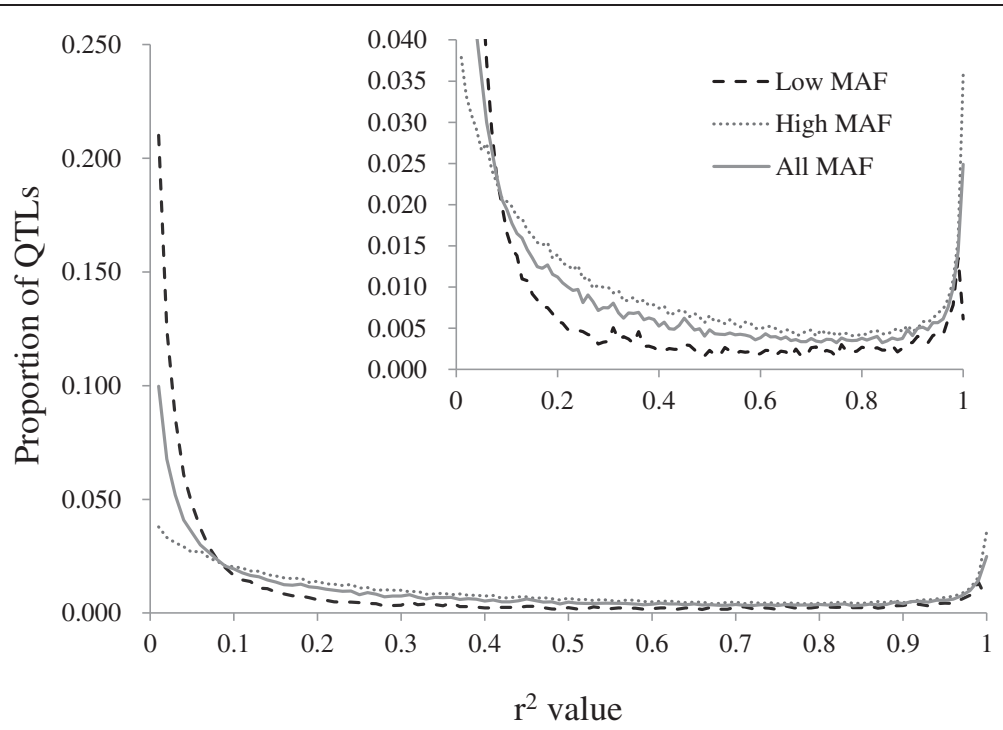

Fig. 2 Proportion of linkage disequilibrium value $\left(r^{2}\right)$ between QTLs and adjacent SNPs. The plot on the right upper corner is the zoomed area of the bigger plot. The $x$-axis indicates the $r^{2}$ value between QTLs and SNPS, and the $y$-axis represents the proportion of QTLs in each minor allele frequency (MAF) category (All, Low, and High). The $r^{2}$ values between QTLs and both adjacent SNPs were calculated, and then the maximum value of $r^{2}$ between two QTL-SNP intervals was chosen to plot in each QTL. The parameters used were the same as those under scenario 1

heritability and correlation remained constant, regardless of the number of QTLs in each MAF category.

For the distribution of QTL effect, the results of the QTLs with high and low MAFs followed a similar trend between the two distribution models, whereas different results were observed between two distribution models in the QTLs with all MAFs. The results of high and all MAFs showed similar trends in the gamma distribution model, and the estimated QTL heritability and correlation between TBV and GEBV were about 0.39 and 0.50 , respectively. On the other hand, the results of all MAFs were lower than those of high MAF in the equal variance model, and the values of estimated QTL heritability and correlation between TBV and GEBV were about 0.36 and 0.44 for all MAF and 0.39 and 0.50 for high MAF, respectively.

\section{The evaluation strategy}

We evaluated the impact of the MAF of QTLs on genomic evaluation under different evaluation strategy for SNP density and prediction model in scenario 3. Goodness-of-fit was measured by the Akaike information criterion (AIC) to compare the prediction models. The AIC is defined as $A I C=2 v-2 \ln ($ likelihood $)$, where $v$ is the number of variance components. This formula shows that the goodness of fit is high, if the AIC is low. The estimated QTL heritability, AIC, and correlation between TBV and GEBV are shown in Table 2, Table 3 , and Table 4, respectively.

Differences in the SNP density have an impact on heritability estimation and GEBV prediction. For model (1) with $G_{Y}$, the results of $50 \mathrm{~K}$ were higher than those of
$7 \mathrm{~K}$ in all MAF categories. For example, from the QTLs with all MAFs, the results of $50 \mathrm{~K}$ and $7 \mathrm{~K}$ were 0.36 and 0.30 for QTL heritability and 0.44 and 0.42 for correlation between TBV and GEBV, respectively. The results of imputed SNPs (i.e., 7 K_to_HD and 50 K_to_HD) were higher than those of $7 \overline{\mathrm{K}}$ and $50 \mathrm{~K}$, and were very close to the results of perfect LD SNPs (i.e., HD) in all MAF categories. For example, from the QTLs with all MAFs, the results of both 50 K_to_HD and 7 K_to_HD were 0.37 for QTL heritability and 0.45 for correlation between TBV and GEBV, and the results of HD were 0.38 for QTL heritability and 0.45 for correlation between TBV and GEBV. These results indicate that heritability estimation and GEBV prediction depend on the SNP density. However, the different results among SNP densities in each MAF category depend on the prediction model.

For the prediction model, the result of model (1) with $\mathrm{G}_{\mathrm{V}}$ was similar to that with $\mathrm{G}_{\mathrm{Y}}$ in the QTL with high MAF, but the difference between the results obtained from $G_{V}$ and $G_{Y}$ increased in the QTL with low MAF. For example, the differences between $G_{V}$ and $G_{Y}$ in the AIC and correlation between TBV and GEBV with $50 \mathrm{~K}$ were 0 and 0.00 in the QTL with high MAF but 7 and 0.03 in the QTL with low MAF, respectively. The result of model (1) with $G_{S}$ was similar to or better than that with $G_{Y}$ in the QTL with all and low MAFs, but performed worse in the QTL with high MAF. In particular, the difference in the results between $G_{S}$ and $G_{Y}$ in the QTL with high MAF was increased at larger SNP density. For example, the difference between $G_{S}$ and $G_{Y}$ in AIC and correlation between TBV and GEBV were 1 

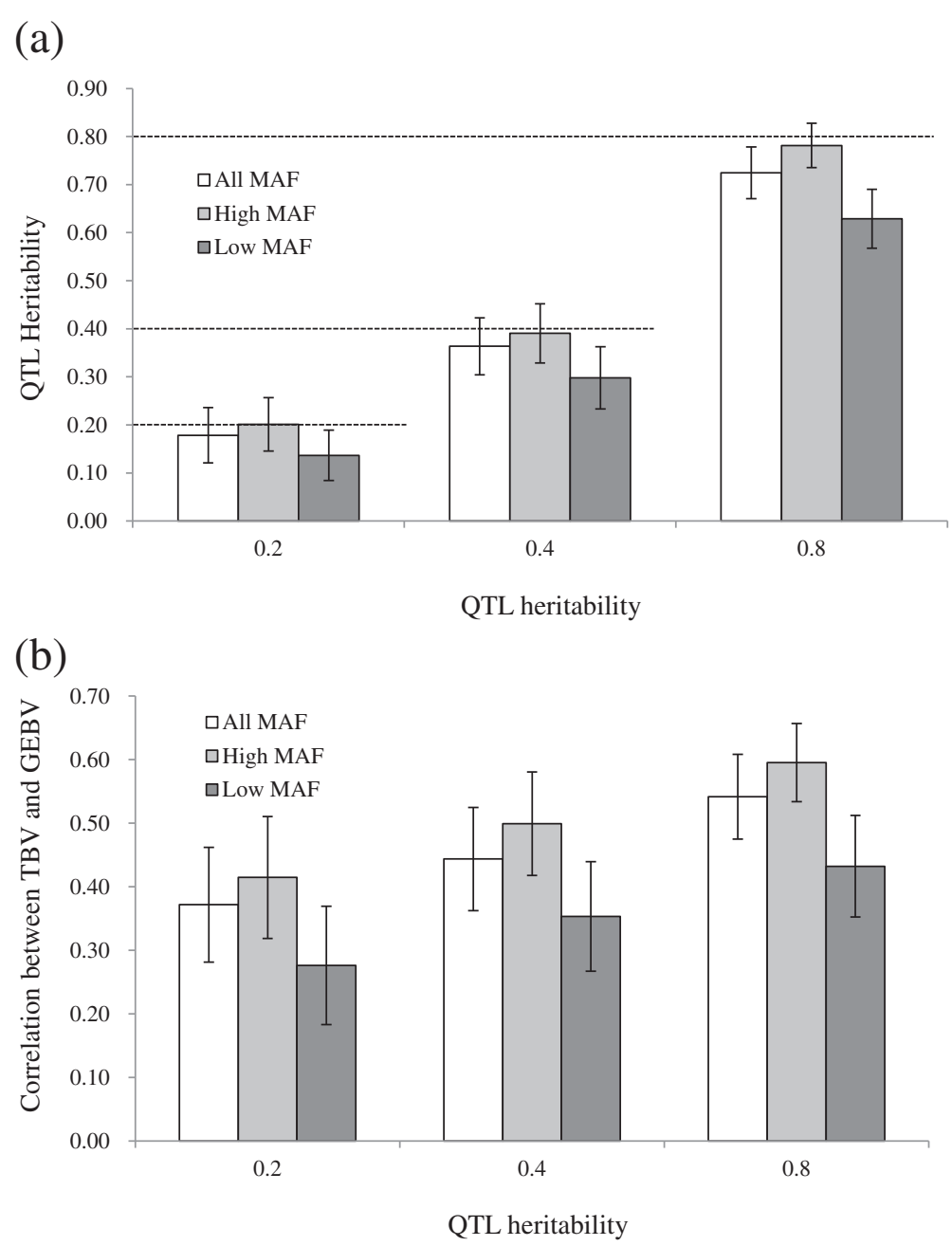

Fig. 3 Results obtained from scenario 1. Estimated QTL heritability and correlation between true breeding and genomic estimated breeding values are calculated. The $x$-axis indicates the true QTL heritability, and the $y$-axis represents mean values of 300 replicates for the estimated QTL heritability (a) and the correlation between true breeding value (TBV) and genomic estimated breeding value (GEBV) (b). The results of varying minor allele frequency (MAF) categories (All, Low, and High) and QTL heritabilities $(0.20,0.40$, and 0.80$)$ are shown. The whiskers represent the standard deviation of 300 replicates

and 0.00 in $7 \mathrm{~K}$ but 10 and 0.03 in $\mathrm{HD}$. In addition, the results of Gs with HD in high MAF were 6147 in AIC and 0.48 in the correlation between TBV and GEBV, which represented the worst of all results by other models under the high MAF scenario. The results of model (2) were similar to or better than those of the other three models under all MAF categories. In particular, the results of model (2) with HD in low MAF, which were 6150 in AIC and 0.47 in correlation between TBV and GEBV, representing the best values in the low MAF results.

\section{Population size}

In this simulation, the impact of the MAF of QTLs on genomic evaluation under different population size was evaluated in scenario 4. The estimated QTL heritability and correlation between TBV and GEBV are shown in
Fig. 5. The results of heritability estimation and GEBV prediction followed a different trend. The mean values of estimated QTL heritability were close to the setting value (0.40) and were almost the same as those among different population sizes, but the SD of the estimated results decreased as the size of the population increased (e.g., from 0.47 to 0.07 in reference size from 200 to 1200 , respectively, for all MAFs). The following trend of the results, the mean values of high MAF > all MAF > low MAF, was shown for QTL heritability, when the size of reference set was more than 800 . These results indicated that the heritability estimates at lower population sizes are less precise than those at higher population sizes, even if the estimated value is close to the setting value. In addition, the impact of the MAF of QTLs was shown at larger population sizes. 

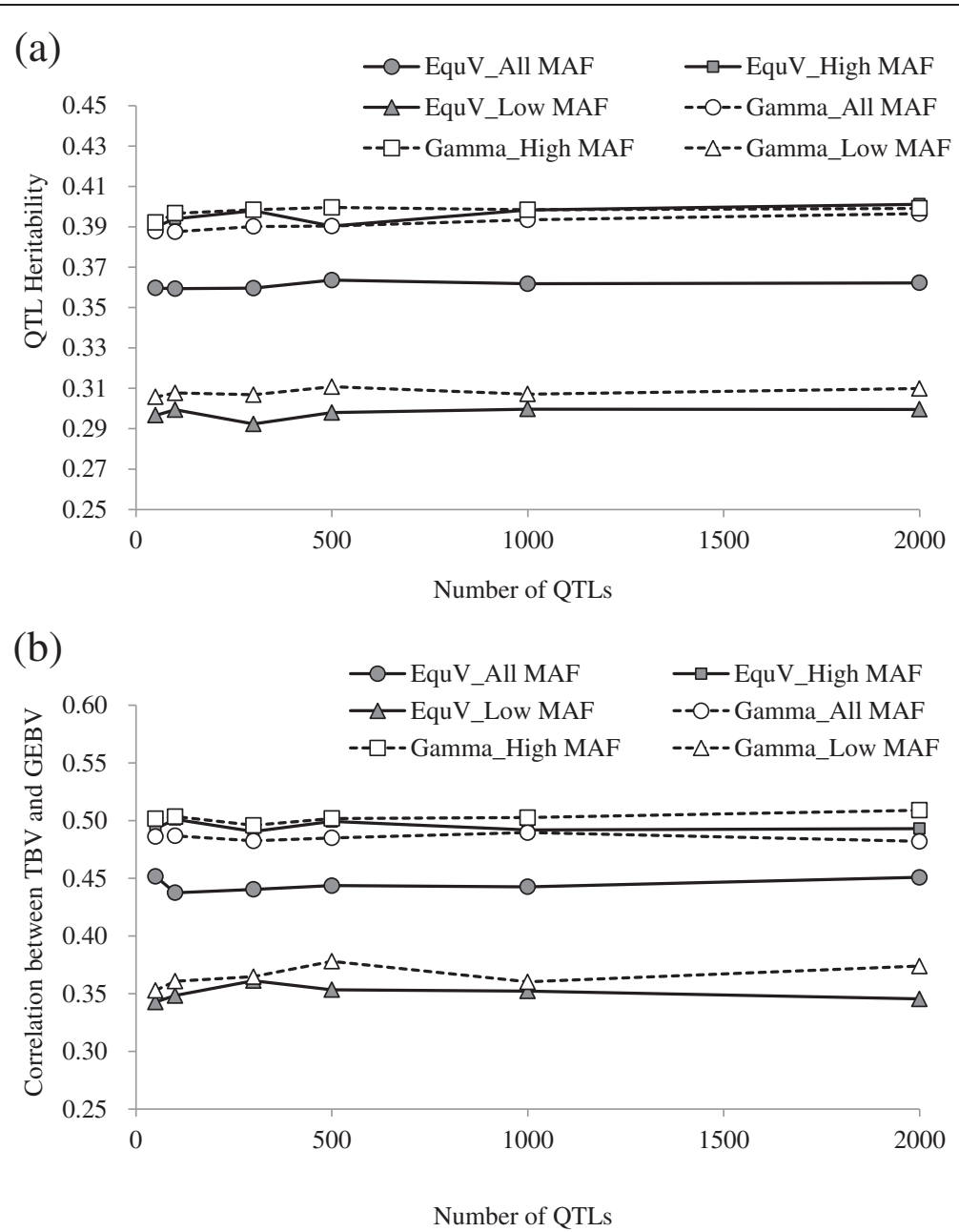

Fig. 4 Results obtained from scenario 2. Estimated QTL heritability and correlation between true breeding and genomic estimated breeding values are calculated. The $x$-axis indicates the number of QTLs, and the $y$-axis represents mean values of 300 replicates for the estimated QTL heritability (a) and the correlation between true breeding value (TBV) and genomic estimated breeding value (GEBV) (b). The results of varying minor allele frequency (MAF) categories (All, Low, and High), number of QTLs (50, 100, 300, 500, 1000, and 2000), and distribution of QTL allele substitution effect (Gamma, gamma distribution model; EquV, equal variance model) are shown

In the GEBV prediction, the correlations between TBV and GEBV were increased as the size of the reference increased (e.g., 0.11-0.41 at reference size 200-1200, respectively, for all MAFs). QTLs with high MAF had the highest value, and the values of all MAFs were between those with low and high MAFs in all reference sizes (e.g., 0.34, 0.41, and 0.50 for low, all, and high MAFs in reference size 1200, respectively). In addition, as the size of the reference increased from 200 to 1200 , the difference between the high and low MAFs for the correlations between TBV and GEBV increased from 0.07 to 0.15 , respectively.

\section{Discussion}

\section{The genetic architecture}

The differences in the QTL heritability and the distribution of QTL effect had an impact on heritability estimation and GEBV prediction under different MAF categories, but the differences in the number of QTL did not have. The results of the correlation between TBV and GEBV for the number of QTL were the same as those described by Daetwyler et al. [10], because the accuracy of GBLUP is constant regardless of the number of QTLs. The trend of the results for QTL heritability was similar to that described by Yang et al. [6].

For the distribution of QTL effect, the genetic variance of the $j$-th QTL is theoretically calculated as $2 p_{j}\left(1-p_{j}\right) \alpha_{j}^{2}$, where $p_{j}$ is the allele frequency of QTLs and $\alpha_{j}$ is the QTL effect [22]. This formula shows that the QTL effect will increase as the allele frequency decreases, if the genetic variance is constant. Therefore, the QTLs with low MAF must have a higher QTL effect than those with high MAF to contribute to the total genetic variance. In a real data analysis, findings from a meta-analysis of human height showed that the QTLs with high MAF had 
Table 2 Heritability estimation in scenario 3

\begin{tabular}{|c|c|c|c|c|c|c|c|}
\hline \multirow[b]{2}{*}{ SNP ${ }^{b}$} & \multirow[b]{2}{*}{ Prediction model $^{c}$} & \multicolumn{2}{|c|}{ All MAF ${ }^{a}$} & \multicolumn{2}{|c|}{ High MAF ${ }^{a}$} & \multicolumn{2}{|c|}{ Low MAF ${ }^{a}$} \\
\hline & & Mean & SD & Mean & SD & Mean & SD \\
\hline \multirow[t]{4}{*}{$7 \mathrm{~K}$} & Model (1) with $G_{V}$ & 0.28 & 0.05 & 0.32 & 0.05 & 0.20 & 0.06 \\
\hline & Model (1) with $G_{Y}$ & 0.30 & 0.05 & 0.33 & 0.05 & 0.23 & 0.06 \\
\hline & Model (1) with $G_{S}$ & 0.30 & 0.05 & 0.33 & 0.05 & 0.24 & 0.06 \\
\hline & Model (2) & 0.30 & 0.05 & 0.33 & 0.05 & 0.23 & 0.06 \\
\hline \multirow[t]{4}{*}{$50 \mathrm{~K}$} & Model (1) with $G_{V}$ & 0.33 & 0.06 & 0.38 & 0.06 & 0.24 & 0.06 \\
\hline & Model (1) with $G_{Y}$ & 0.36 & 0.06 & 0.39 & 0.06 & 0.30 & 0.06 \\
\hline & Model (1) with $G_{S}$ & 0.38 & 0.06 & 0.40 & 0.06 & 0.34 & 0.07 \\
\hline & Model (2) & 0.37 & 0.06 & 0.39 & 0.06 & 0.34 & 0.06 \\
\hline \multirow[t]{4}{*}{ 7K_to_HD } & Model (1) with $G_{V}$ & 0.34 & 0.06 & 0.39 & 0.06 & 0.24 & 0.06 \\
\hline & Model (1) with $G_{Y}$ & 0.37 & 0.06 & 0.40 & 0.06 & 0.30 & 0.06 \\
\hline & Model (1) with $G_{S}$ & 0.41 & 0.07 & 0.41 & 0.07 & 0.39 & 0.07 \\
\hline & Model (2) & 0.39 & 0.06 & 0.40 & 0.06 & 0.38 & 0.06 \\
\hline \multirow[t]{4}{*}{ 50K_to_HD } & Model (1) with $G_{V}$ & 0.34 & 0.06 & 0.39 & 0.06 & 0.25 & 0.06 \\
\hline & Model (1) with $G_{Y}$ & 0.37 & 0.06 & 0.41 & 0.06 & 0.30 & 0.07 \\
\hline & Model (1) with $G_{S}$ & 0.41 & 0.07 & 0.42 & 0.07 & 0.40 & 0.07 \\
\hline & Model (2) & 0.40 & 0.06 & 0.40 & 0.06 & 0.40 & 0.06 \\
\hline \multirow[t]{4}{*}{$\mathrm{HD}$} & Model (1) with $G_{V}$ & 0.35 & 0.06 & 0.39 & 0.06 & 0.25 & 0.06 \\
\hline & Model (1) with $G_{Y}$ & 0.38 & 0.06 & 0.41 & 0.06 & 0.31 & 0.07 \\
\hline & Model (1) with $G_{S}$ & 0.42 & 0.07 & 0.41 & 0.07 & 0.40 & 0.07 \\
\hline & Model (2) & 0.40 & 0.06 & 0.40 & 0.06 & 0.41 & 0.06 \\
\hline
\end{tabular}

${ }^{a} \mathrm{MAF}$, Minor allele frequency; All MAF, $0.01 \leq \mathrm{MAF} \leq 0.5$; High MAF, $0.05<$ $\mathrm{MAF} \leq 0.5$; Low $\mathrm{MAF}, 0.01 \leq \mathrm{MAF} \leq 0.05$

${ }^{\mathrm{b}} 7 \mathrm{~K}, 50 \mathrm{~K}$ and HD, Illumina infinium BovineLDv1.1, BovineSNP50v2, and BovineHD BeadChips, respectively; 7 K_to_HD and 50 K_to_HD, Imputations were performed from $7 \mathrm{~K}$ and $50 \mathrm{~K}$ to $\mathrm{HD}$, respectively

${ }^{\prime} \mathrm{G}_{\mathrm{V}}$, VanRaden's genome relationship matrix (GRM); $\mathrm{G}_{\mathrm{Y}}$, Yang's GRM; $\mathrm{G}_{\mathrm{s}}$ Speed's GRM

small phenotypic effects, whereas the QTLs with low MAF had large effects on this trait such as a function of the MAF [23]. Therefore, missing heritability has focused on the possible contribution of QTLs with low MAF, and the QTLs with low MAF could have an intermediate effect [24]. In this study, the factor for the distribution of QTL effect was evaluated to account for the low-MAF QTL with intermediate effect. In the gamma distribution model, the low-MAF QTL with intermediate effect cannot be defined in the QTL with all MAFs, because the QTL effect was randomly allocated to the MAF. On the other hand, this QTL can be defined in the equal variance model. As an example, the mean values for the QTL effect and QTL variance for the QTL with all MAFs as a function of MAF are shown in Additional file 1: Figure S2. Additional file 1: Figure S2 is drawn from a result of the randomly selected replica under scenario 2 with the parameters for the number of QTLs (500). In this result, no relationship between MAF, QTL effect, and QTL variance was observed in the gamma distribution model, whereas the QTL with low MAF had a higher QTL
Table 3 Model fitness measured by Akaike information criterion (AIC) in scenario 3

\begin{tabular}{|c|c|c|c|c|c|c|c|}
\hline \multirow[b]{2}{*}{ SNP ${ }^{b}$} & \multirow[b]{2}{*}{ Prediction model ${ }^{c}$} & \multicolumn{2}{|c|}{ All $M_{A} F^{a}$} & \multicolumn{2}{|c|}{ High $\mathrm{MAF}^{a}$} & \multicolumn{2}{|c|}{ Low MAF ${ }^{a}$} \\
\hline & & Mean & $\overline{S D}$ & $\overline{\text { Mean }}$ & $\overline{S D}$ & Mean & $\mathrm{SD}$ \\
\hline \multirow[t]{4}{*}{$7 \mathrm{~K}$} & Model (1) with $G_{V}$ & 6164 & 63 & 6145 & 66 & 6191 & 61 \\
\hline & Model (1) with $G_{Y}$ & 6162 & 63 & 6145 & 66 & 6188 & 61 \\
\hline & Model (1) with $G_{S}$ & 6162 & 63 & 6146 & 66 & 6187 & 61 \\
\hline & Model (2) & 6163 & 63 & 6147 & 66 & 6186 & 61 \\
\hline \multirow[t]{4}{*}{$50 \mathrm{~K}$} & Model (1) with $G_{V}$ & 6159 & 63 & 6139 & 65 & 6188 & 62 \\
\hline & Model (1) with $G_{Y}$ & 6155 & 63 & 6139 & 65 & 6181 & 62 \\
\hline & Model (1) with $G_{s}$ & 6155 & 63 & 6142 & 65 & 6175 & 62 \\
\hline & Model (2) & 6155 & 63 & 6140 & 65 & 6163 & 62 \\
\hline \multirow[t]{4}{*}{ 7K_to_HD } & Model (1) with $G_{V}$ & 6158 & 63 & 6138 & 65 & 6189 & 62 \\
\hline & Model (1) with $G_{Y}$ & 6155 & 63 & 6138 & 65 & 6182 & 62 \\
\hline & Model (1) with Gs & 6156 & 63 & 6147 & 65 & 6171 & 62 \\
\hline & Model (2) & 6154 & 63 & 6139 & 65 & 6155 & 62 \\
\hline \multirow[t]{4}{*}{ 50K_to_HD } & Model (1) with $G_{V}$ & 6157 & 63 & 6137 & 65 & 6188 & 62 \\
\hline & Model (1) with $G_{Y}$ & 6154 & 63 & 6137 & 65 & 6181 & 62 \\
\hline & Model (1) with $G_{S}$ & 6155 & 63 & 6146 & 65 & 6169 & 62 \\
\hline & Model (2) & 6153 & 63 & 6138 & 65 & 6152 & 62 \\
\hline \multirow[t]{4}{*}{$H D$} & Model (1) with $G_{V}$ & 6157 & 63 & 6136 & 65 & 6188 & 62 \\
\hline & Model (1) with $G_{Y}$ & 6154 & 63 & 6137 & 65 & 6180 & 62 \\
\hline & Model (1) with $G_{S}$ & 6155 & 63 & 6147 & 65 & 6168 & 62 \\
\hline & Model (2) & 6152 & 63 & 6138 & 65 & 6150 & 62 \\
\hline
\end{tabular}

${ }^{a} \mathrm{MAF}$, Minor allele frequency; All MAF, $0.01 \leq \mathrm{MAF} \leq 0.5$; High MAF, $0.05<$ $\mathrm{MAF} \leq 0.5$; Low MAF, $0.01 \leq \mathrm{MAF} \leq 0.05$

${ }^{b_{7 K}}, 50 \mathrm{~K}$ and HD, Illumina infinium BovineLDv1.1, BovineSNP50v2, and BovineHD BeadChips, respectively; $7 \mathrm{~K}$ _to_HD and $50 \mathrm{~K}$ _to_HD, Imputations were performed from $7 \mathrm{~K}$ and $50 \mathrm{~K}$ to $\mathrm{HD}$, respectively

${ }^{\prime} G_{v}$, VanRaden's genome relationship matrix (GRM); $G_{Y}$, Yang's GRM; $G_{s}$, Speed's GRM

effect and all QTLs had equal genetic variance in the equal variance model. Therefore, the results of the QTLs with all and high MAFs in Fig. 4 showed the same as that under the gamma distribution model, because of the low contribution of the QTLs with low MAF on the total genetic variance. This result was the similar trend as described by Wientjes et al. [25]. This result also shows that the equal variance model accounts for missing heritability in a simulation when the QTLs are composed of variants with low and high MAFs. If QTLs with a large effect do exist, they are at a low frequency and individually explain a small proportion of genetic variance [26]. Therefore, the equal variance model was used to evaluate the impact of MAF of QTL in all scenarios.

\section{The evaluation strategy}

In this study, three types of SNPs were assumed: the imperfect LD SNPs (7 K and $50 \mathrm{~K}$ ), the imputed SNPs (7 K_to_HD and $\left.50 \mathrm{~K} \_t o \_H D\right)$, and the perfect LD SNPs 
Table 4 Correlation between true breeding value and genomic breeding value in scenario 3

\begin{tabular}{|c|c|c|c|c|c|c|c|}
\hline \multirow[b]{2}{*}{ SNP ${ }^{b}$} & \multirow[b]{2}{*}{ Prediction model ${ }^{c}$} & \multicolumn{2}{|c|}{ All $M_{A} F^{a}$} & \multicolumn{2}{|c|}{ High $\mathrm{MAF}^{\mathrm{a}}$} & \multicolumn{2}{|c|}{ Low MAF ${ }^{a}$} \\
\hline & & Mean & SD & Mean & SD & Mean & SD \\
\hline \multirow[t]{4}{*}{$7 \mathrm{~K}$} & Model (1) with $G_{V}$ & 0.41 & 0.08 & 0.48 & 0.08 & 0.30 & 0.09 \\
\hline & Model (1) with $G_{Y}$ & 0.42 & 0.08 & 0.48 & 0.08 & 0.32 & 0.09 \\
\hline & Model (1) with $G_{S}$ & 0.42 & 0.08 & 0.48 & 0.08 & 0.33 & 0.09 \\
\hline & Model (2) & 0.42 & 0.08 & 0.48 & 0.08 & 0.33 & 0.09 \\
\hline \multirow[t]{4}{*}{$50 \mathrm{~K}$} & Model (1) with $G_{V}$ & 0.43 & 0.08 & 0.50 & 0.08 & 0.32 & 0.09 \\
\hline & Model (1) with $G_{Y}$ & 0.44 & 0.08 & 0.50 & 0.08 & 0.35 & 0.09 \\
\hline & Model (1) with $G_{S}$ & 0.44 & 0.08 & 0.49 & 0.08 & 0.37 & 0.09 \\
\hline & Model (2) & 0.44 & 0.08 & 0.50 & 0.08 & 0.41 & 0.09 \\
\hline \multirow[t]{4}{*}{ 7K_to_HD } & Model (1) with $G_{V}$ & 0.44 & 0.08 & 0.50 & 0.08 & 0.32 & 0.09 \\
\hline & Model (1) with $G_{Y}$ & 0.45 & 0.08 & 0.50 & 0.08 & 0.35 & 0.09 \\
\hline & Model (1) with $G_{s}$ & 0.44 & 0.08 & 0.48 & 0.08 & 0.38 & 0.08 \\
\hline & Model (2) & 0.45 & 0.08 & 0.50 & 0.08 & 0.44 & 0.08 \\
\hline \multirow[t]{4}{*}{ 50K_to_HD } & Model (1) with $G_{V}$ & 0.44 & 0.08 & 0.51 & 0.08 & 0.32 & 0.09 \\
\hline & Model (1) with $G_{Y}$ & 0.45 & 0.08 & 0.51 & 0.08 & 0.36 & 0.09 \\
\hline & Model (1) with $G_{S}$ & 0.44 & 0.08 & 0.48 & 0.08 & 0.39 & 0.08 \\
\hline & Model (2) & 0.46 & 0.08 & 0.51 & 0.08 & 0.46 & 0.08 \\
\hline \multirow[t]{4}{*}{ HD } & Model (1) with $G_{V}$ & 0.44 & 0.08 & 0.51 & 0.08 & 0.32 & 0.09 \\
\hline & Model (1) with $G_{Y}$ & 0.45 & 0.08 & 0.51 & 0.08 & 0.36 & 0.08 \\
\hline & Model (1) with $G_{s}$ & 0.44 & 0.08 & 0.48 & 0.08 & 0.39 & 0.08 \\
\hline & Model (2) & 0.46 & 0.08 & 0.51 & 0.08 & 0.47 & 0.08 \\
\hline
\end{tabular}

${ }^{a} \mathrm{MAF}$, Minor allele frequency; All MAF, $0.01 \leq \mathrm{MAF} \leq 0.5$; High MAF, $0.05<$ $\mathrm{MAF} \leq 0.5$; Low MAF, $0.01 \leq \mathrm{MAF} \leq 0.05$

${ }^{{ }^{7}} 7 \mathrm{~K}, 50 \mathrm{~K}$ and HD, Illumina infinium BovineLDv1.1, BovineSNP50v2, and BovineHD BeadChips, respectively; $7 \mathrm{~K}$ to $\mathrm{HD}$ and $50 \mathrm{~K}$ to $\mathrm{HD}$, Imputations were performed from $7 \mathrm{~K}$ and $50 \mathrm{~K}$ to $\mathrm{HD}$, respectively

${ }^{\mathrm{C}} \mathrm{G}_{\mathrm{V}}$, VanRaden's genome relationship matrix (GRM); $\mathrm{G}_{\mathrm{Y}}$, Yang's GRM; $\mathrm{G}_{\mathrm{s}}$ Speed's GRM

(HD). Recently, WGS data are becoming increasingly available for use in cattle, and the 1000 bull genomes project provides annotated sequence variants and genotypes of key ancestor bulls [3]. One of the major advantages of WGS data is that they provide complete information on all the variants of an individual, which include many of the SNPs with low MAF that are not covered by the SNP array. Most of the low MAF variants are only accessible through WGS data, and this information could be important for genomic evaluation. WGS data can be obtained directly by next-generation sequencing techniques or indirectly by genotype imputation. When using imputed SNPs, the impact of imputation error on genomic evaluation must be investigated in genotype imputation. Therefore, the imputed SNPs (indirect information) and the perfect LD SNPs (direct information) were used to evaluate the effectiveness of using WGS data directly or indirectly. The results showed that differences in the SNP density have an impact on heritability estimation and GEBV prediction, especially in the low MAF scenario. For the imperfect LD SNPs, the distribution of MAF for $7 \mathrm{~K}$ followed a different trend compared to HD. On the other hand, the distribution of MAF for $50 \mathrm{~K}$ had the different values but followed a similar trend compared to that for HD, especially at high MAF. Usually, all classes of MAFs are equally represented on a low density SNP array, while the low MAF class is overrepresented in the WGS data [27]. The difference in MAF distribution between QTL and SNPs indicates the difficulty of capturing genetic variance. Therefore, the results of $7 \mathrm{~K}$ were lower than those of $50 \mathrm{~K}$. For the imputed SNPs and the complete LD SNPs, these results were higher than those with $7 \mathrm{~K}$ and $50 \mathrm{~K}$ in heritability estimation and GEBV prediction. The results of imputed SNPs were very close to those of the complete LD SNPs, even if the imputed SNPs were not in perfect LD with the QTL. The number of missing genotypes affects the accuracy, and the difference in imputation accuracy is larger at low MAFs [13]. However, our results showed that there was little difference in the results between $7 \mathrm{~K}$ _to_HD and $50 \mathrm{~K}$ to_HD under the low MAF scenario. A previous study reported that the accuracy of GEBV plateaus on increasing the number of SNPs [12]. On the other hand, GEBV prediction can achieve moderately high prediction accuracy under perfect LD between SNPs and QTLs in distantly related human data [28]. Therefore, using the SNPs related to QTLs directly or indirectly is effective for performing heritability estimation and GEBV prediction.

In this study, we showed that the differences of the result among SNP densities in each MAF category depend on the prediction model. For model (1) with $G_{V}$ and $G_{Y}$, the difference of the results between $G_{V}$ and $G_{Y}$ was increased in the QTL with low MAF. Meuwissen et al. [29] suggested that weighted GRM by MAF would have a better result than unweighted GRM, when a high proportion of loci with low MAF are used. $G_{Y}$ is corrected for variance of the allele frequency of each SNP, and gives weight to alleles with low MAF. On the other hand, $G_{V}$ is corrected for the average frequency of heterozygotes, and gives less weight to alleles with low MAF. Therefore, the approach of $G_{Y}$ was better than that of $G_{V}$, especially under the low MAF scenario. For the model (1) with $G_{S}$ reflecting the degree of $L D$, the difference of the results between $G_{S}$ and $G_{Y}$ in the QTL with high MAF was increased at larger SNP densities, and the result using HD was the worse than that by other prediction models under the high MAF scenario. Lee et al. [30] reported that $G_{S}$ generates biased heritability estimates through the use of denser SNPs, because of too much weight being attributed to the low MAF SNPs. This method accounts for the different extents of LD among SNPs, and weighted SNPs depend on the MAF distribution of SNPs. The distribution of MAF is different between the dense and sparse SNP data, because the 

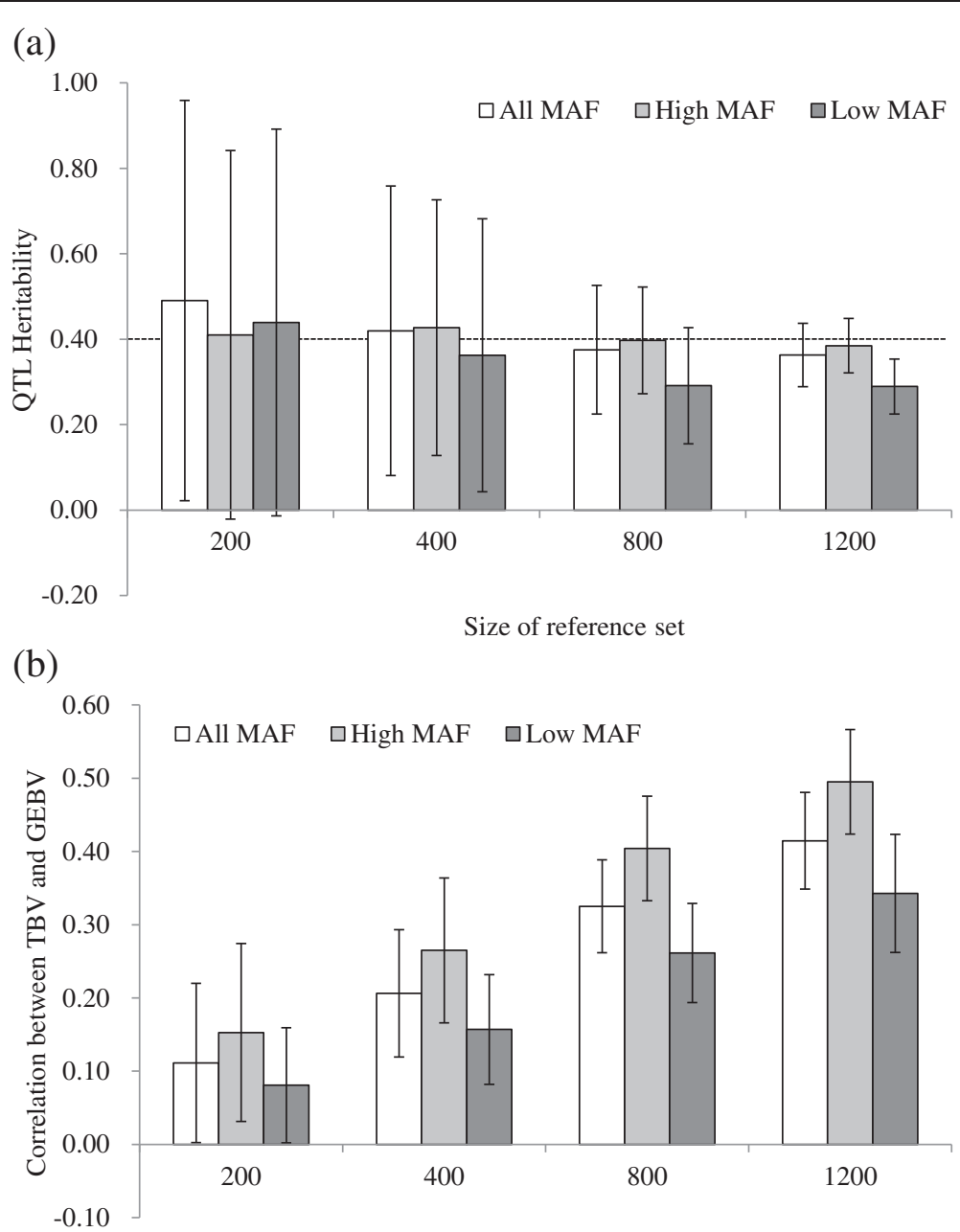

Size of reference set

Fig. 5 Results obtained from scenario 3. Estimated QTL heritability and correlation between true breeding and genomic estimated breeding values are calculated. The $x$-axis indicates the size of the reference set, and the $y$-axis represents mean values of 300 replicates for the estimated QTL heritability (a) and the correlation between true breeding value (TBV) and genomic estimated breeding value (GEBV) (b). The results of varying minor allele frequency (MAF) categories (All, Low, and High) and size of the reference set (200,400, 800, and 1200) are shown. The whiskers represent the standard deviation of 300 replicates

proportion of SNPs with low MAF increased as SNP density increased. The low MAF SNPs could be under low LD among SNPs and the proportion of weighted SNPs with low MAF will increase. In this simulation, the proportion of SNPs before and after weighting for $\mathrm{G}_{\mathrm{S}}$ is different for sparse and dense SNP data, and the proportion of weighted SNPs with low MAF was higher than that with high MAF in imputed SNPs and perfect LD SNPs (Additional file 2: Table S1). Therefore, the result of the QTL with high MAF was the lowest, because many of the low MAF SNPs were weighted in $\mathrm{G}_{\mathrm{S}}$. To account for the degree of LD between SNPs at larger SNP densities, the haplotype model (such as Sun et al. [31]) could have significant average in low MAF QTL.

For model (2), the results were similar to or better than those from the other three models under all MAF categories, and represented the best value when a higher proportion of QTLs that had low MAF and SNPs on HD were used. This model means that QTL heritability is partitioned by the MAF of SNPs to provide insight into genetic architecture. Some studies have showed that different MAF categories could deliver estimates with little bias and high goodness-of-fit in human $[30,32]$ and chicken population [33,34], because high LD is only possible between SNPs with similar MAFs. In addition, Lee et al. [30] also showed that the different MAF categories generate heritability estimates with higher goodness-of-fit in dense SNP data compared with sparse SNP data, especially when a higher proportion of QTLs have low MAF. In principle, fitting more MAF categories in a prediction model could more accurately represent the genetic architecture, but it brings the disadvantage of estimating more 
parameters. Therefore, Lee et al. [30] used five bins with MAF boundaries $0.1,0.2,0.3,0.4$, and 0.5 . In this study, two MAF categories (high and low) were fitted in the model (2), because the QTL can be roughly classified into two types: an intermediate effect at low MAF and a small effect at high MAF under missing heritability. Therefore, more MAF categories were not fitted at high MAF. Our results using model (2) with HD were better than those based on five MAF categories in all MAF categories of this simulation (Additional file 2: Table S2). Therefore, model (2) is a robust method in all MAF categories and could capture more of the genetic variance under the low MAF scenario if the WGS data are available.

\section{Population size}

In this simulation, a high proportion of genetic variance was captured in the high MAF scenario. However, our results did not reflect high precision, and the accuracy of GEBV remained lower than that of the simulation study [2], even if the perfect LD SNP was used under the high MAF scenario. Under this simulation, the maximum correlation between TBV and GEBV for the QTLs with all, high, and low MAFs was $0.46,0.51$, and 0.47 , respectively, for QTL heritability of 0.40 . The main reason for low prediction accuracy could be the size of the reference population. The accuracy of GEBV depends on the size of the reference, and a large sample of animals is needed in the reference population if accurate GEBV prediction is desired [1]. Daetwyler et al. [35] also described the accuracy of GEBV deterministically as follows:

$$
r=\sqrt{\frac{N h^{2}}{N h^{2}+q}}
$$

where $N$ is the number of individuals in the reference population, $h^{2}$ is heritability, and $q$ is the number of independent loci affecting a trait. This formula shows that the accuracy of GEBV depends on the size of the reference and the number of QTLs. Daetwyler et al. [35] also showed that GBLUP has a constant accuracy for a given $N$ and $h^{2}$, regardless of $q$. Therefore, the accuracy of GEBV increases with a larger reference size.

In this study, the accuracy increased as the size of the reference increased in all MAF categories, and the accuracy did not plateau at the maximum size of the reference (Fig. 5). Under this simulation, there were insufficient DNA samples to evaluate the impact of a larger sample size. Therefore, further study is needed to evaluate the impact of population size on genomic prediction under different MAF scenarios.

\section{Simulation based on real data}

Populations containing related individuals (e.g., cattle) are expected to yield high LD between SNPs and QTLs than populations containing unrelated individuals (e.g., human), because of decreasing effective population size. The LD in cattle follows a similar pattern to that of humans at short distances, but is much larger than that of humans at long distances [1]. The extent of LD between SNPs and QTLs depends on a population structure, and the impact of MAF of QTL must be evaluated by the use of datasets accounting for the extent of LD in a target population. The effective population size of Japanese Black cattle has been reduced because of the intensive use of a few sires with high marbling [36], and the extent of LD could be higher than that in other cattle breeds. There has been little assessment of the MAF of QTLs that affect heritability estimation and GEBV prediction using real cattle data except for Wientjes et al. [25] in Dairy cattle population. Therefore, we used real genotype data in the simulation study, which accounted for the extent of LD in Japanese Black cattle. For the extent of LD in this target population, the extent of LD between SNPs and QTLs increased as the MAF of QTL increased in the scenario with $50 \mathrm{~K}$. The main reason was that $87 \%$ of SNPs in $50 \mathrm{~K}$ were high MAF (see Additional file 2: Table S1). On the other hand, only $13 \%$ of SNPs were low MAF, and most of QTLs with low MAF were in low LD with SNPs in $50 \mathrm{~K}$. This indicates that the concordance of MAF between QTL and SNP shows higher LD between two loci in this population.

Under this simulation, we showed that the results of these predicted models with different SNP densities depend on the genetic architecture of objective traits, especially the MAF of QTLs. Recently, some studies have investigated the proportion of genetic variance captured by SNPs and the prediction accuracy of GEBV in small Japanese Black cattle populations using the GBLUP method with $\mathrm{G}_{\mathrm{V}}$ and $50 \mathrm{~K}$ [37-39]. In those studies, a higher proportion of genetic variance was captured for carcass traits, and these proportions were close to the genetic variance previously reported by estimations based on pedigree information. In our results with the scenario of all MAF category and setting QTL heritability $(0.40)$, the genome heritability was 0.33 for the scenario with $G_{V}$ and $50 \mathrm{~K}$, whereas there was genome heritability of 0.40 for the scenario with model (2) and HD. In addition, previous studies show that the some proportion of the genetic variance are not captured by SNPs with high MAF in Holstein cattle population [40, 41] and in chicken population [33]. Therefore, the explanation of a high proportion may be that there are not only the QTLs with high MAF but also strong relationship structures in these populations, because our population was excluded very close relatives to obtain low relationship structure. The strong relationship structures also lead to capture more of genetic variance by SNPs with high MAF. To evaluate the genetic architecture of 
complex traits, we suggest that it is effective to compare among these prediction models with different SNP densities. However, it is not sufficient for the evaluation of predictive ability, because of the difficulty of obtaining TBV. It may not reflect the predictive ability, even if a higher proportion of genetic variance is captured. In this population, the extent of LD was still higher than that in other beef breeds [13], even if we excluded very close relatives to evaluate the minimum value of QTL heritability and correlation in Japanese Black cattle. These results show that a higher proportion of genetic variance was captured under the high MAF scenario. However, the accuracy of GEBV remained low, and the goodness-of-fit did not increase the prediction accuracy. Therefore, further study including additional animals in the reference population is needed to increase the prediction accuracy.

\section{Conclusions}

The current study evaluated the impact of MAF of QTL on genomic evaluation in a simulation study by assuming different MAFs of QTLs and several factors in Japanese Black cattle. The extent of LD between SNPs and QTLs was higher in the QTLs with high MAF than in those with low MAF. The MAF of QTLs had an impact on heritability estimation and prediction accuracy and that depended on the genetic architecture, evaluation strategy and population size. The genetic architecture results showed that genomic evaluation was affected by the MAF of QTLs combined with the QTL heritability and the distribution of QTL effect. The number of QTL was not affected on genomic evaluation if the number of QTL was more than 50. For the evaluation strategy, different SNP densities and prediction models affected the heritability estimation and genomic prediction, and these depended on the MAF of QTLs. The genetic dissection of complex traits would be possible by comparing the results of these predicted models with different SNP densities. In addition, accurate QTL heritability and GEBV were obtained by using denser SNP information and model (2) under all MAF categories. However, it may not reflect the predictive ability, and a larger sample size is needed to increase the accuracy of GEBV.

\section{Availability of supporting data}

The data sets supporting the results of this article are included within the article and its additional file.

\section{Additional files}

Additional file 1: Figure S1. Distribution of progenies per sire in this population. The $x$-axis indicates the number of progenies per sire, and the $y$-axis represents the number of sires. Figure S2. QTL effect and QTL variance as a function of minor allele frequency (MAF). The $x$-axis indicates the MAF of SNPs, and the $y$-axis represents the QTL effect (a) and QTL variance (b) in a randomly selected replica. The results of varying distributions of QTL allele substitution effects (Gamma, gamma distribution model; EquV, equal variance model) for all MAF, QTL heritability (0.40), and the number of QTLs (500) are shown. (PPTX 51 kb)

Additional file 2: Table S1. Number of SNPs before and after weighting for Speed's genomic relationship matrix. Table S2. Comparison of two and five minor allele frequency (MAF) categories. (DOC $62 \mathrm{~kb}$ )

\section{Abbreviations}

AIC: Akaike information criterion; GBLUP: Genomic best linear unbiased prediction; GEBV: Genomic estimated breeding value; GRM: Genomic relationship matrix; LD: Linkage disequilibrium; MAF: Minor allele frequency; QTL: Quantitative trait locus; SNP: Single nucleotide polymorphism; TBV: True breeding value; WGS: Whole genome sequence.

\section{Competing interests}

The authors declare that they have no competing interests.

\section{Authors' contributions}

YU participated in the design of the study, performed the statistical analysis, and drafted the manuscript. SS, YS and TW participated in the design of the study, provided the raw data, and contributed in writing and improving the manuscript. TK participated in the design of the study, and contributed in writing and improving the manuscript. All authors read and approved the final manuscript.

\section{Acknowledgements}

The authors thank the staff of the Shirakawa Institute of Animal Genetics for technical assistance. This work was supported by the Japan Racing and Livestock Promotion to YS.

\section{Author details}

${ }^{1}$ National Livestock Breeding Center, Nishigo, Fukushima 961-8511, Japan.

${ }^{2}$ Shirakawa Institute of Animal Genetics, Japan Livestock Technology

Association, Nishigo, Fukushima 961-8511, Japan.

Received: 29 July 2015 Accepted: 27 October 2015

Published online: 19 November 2015

\section{References}

1. Goddard ME, Hayes BJ. Mapping genes for complex traits in domestic animals and their use in breeding programmes. Nat Rev Genet. 2009;10:381-91.

2. Meuwissen THE, Hayes BJ, Goddard ME. Prediction of total genetic value using genome-wide dense marker maps. Genetics. 2001;157:1819-29.

3. Daetwyler HD, Capitan A, Pausch H, Stothard P, Van Binsbergen R, Brøndum RF, et al. Whole-genome sequencing of 234 bulls facilitates mapping of monogenic and complex traits in cattle. Nat Genet. 2014;46:858-67.

4. Meuwissen THE, Goddard M. Accurate prediction of genetic values for complex traits by whole-genome resequencing. Genetics. 2010;185:623-31.

5. Maher B. Personal genomes: the case of missing heritability. Nature. 2008;456:18-21.

6. Yang J, Benyamin B, McEvoy BP, Gordon S, Henders AK, Nyholt DR, et al. Common SNPs explain a large proportion of the heritability for human height. Nat Genet. 2010;42:565-9.

7. Nishimura S, Watanabe T, Ogino A, Shimizu K, Morita M, Sugimoto Y, et al. Application of highly differentiated SNPs between Japanese Black and Holstein to a breed assignment test between Japanese Black and F1 (Japanese Black x Holstein) and Holstein. Anim Sci J. 2013;84:1-7.

8. Dekkers JC. Prediction of response to marker-assisted and genomic selection using selection index theory. J Anim Breed Genet. 2007;124:331-41.

9. Makowsky R, Pajewski NM, Klimentidis YC, Vazquez Al, Duarte CW, Allison $D B$, et al. Beyond missing heritability: prediction of complex traits. PLoS Genet. 2011;7:e1002051.

10. Daetwyler HD, Pong-Wong R, Villanueva B, Woolliams JA. The impact of genetic architecture on genome-wide evaluation methods. Genetics. 2010;185:1021-31.

11. Goddard ME. Genomic selection: prediction of accuracy and maximization of long term response. Genetica. 2009;136:245-57. 
12. Hayes BJ, Bowman PJ, Chamberlain AJ, Goddard ME. Invited review: genomic selection in dairy cattle: progress and challenge. J Dairy Sci. 2009;92:433-43.

13. Uemoto Y, Sasaki S, Sugimoto Y, Watanabe T. Accuracy of high-density genotype imputation in Japanese Black cattle. Anim Genet. 2015;46:388-94.

14. Nishimura S, Watanabe T, Mizoshita K, Tatsuda K, Fujita T, Watanabe N, et al. Genome-wide association study identified three major QTL for carcass weight including the PLAG1-CHCHD7 QTN for stature in Japanese Black cattle. BMC Genet. 2012;13:40.

15. Sasaki S, Ibi T, Watanabe T, Matsuhashi T, Ikeda S, Sugimoto $Y$. Variants in the 3'UTR of General Transcription Factor IIF, polypeptide 2 affect female calving efficiency in Japanese Black cattle. BMC Genet. 2013;14:41.

16. Browning BL, Browning SR. Improving the accuracy and efficiency of identity-by-descent detection in population data. Genetics. 2013;194:459-71.

17. Hill WG, Goddard ME, Visscher PM. Data and theory point to mainly additive genetic variance for complex traits. PLoS Genet. 2008;4:e1000008.

18. VanRaden PM. Efficient methods to compute genomic predictions. J Dairy Sci. 2008;91:4414-23.

19. Speed D, Hemani G, Johnson MR, Balding DJ. Improved heritability estimation from genome-wide SNPs. Am J Hum Genet. 2012;91:1011-21.

20. Gilmour AR, Gogel BJ, Cullis BR, Thompson R. ASRreml User Guide Release 3.0. 2009

21. Purcell S, Neale B, Todd-Brown K, Thomas L, Ferreira MA, Bender D, et al. PLINK: A tool set for whole-genome association and population-based linkage analyses. Am J Hum Genet. 2007:81:559-75.

22. Falconer DS, Mackay TF. Introduction to quantitative genetics. 4th ed. Harlow. UK: Longman; 1996.

23. Lanktree MB, Guo Y, Murtaza M, Glessner JT, Bailey SD, Onland-Moret NC, et al. Meta-analysis of dense genecentric association studies reveals common and uncommon variants associated with height. Am J Hum Genet. 2011;88:6-18.

24. Manolio TA, Collins FS, Cox NJ, Goldstein DB, Hindorff LA, Hunter DJ, et al. Finding the missing heritability of complex diseases. Nature. 2009;461:747-53.

25. Wientjes YC, Calus MP, Goddard ME, Hayes BJ. Impact of QTL properties on the accuracy of multi-breed genomic prediction. Genet Sel Evol. 2015;47:42.

26. Hayes BJ, Pryce J, Chamberlain AJ, Bowman PJ, Goddard ME. Genetic architecture of complex traits and accuracy of genomic prediction: coat colour, milk-fat percentage, and type in Holstein cattle as contrasting model traits. PLoS Genet. 2010;6:e1001139.

27. Eynard SE, Windig JJ, Leroy G, van Binsbergen R, Calus MPL. The effect of rare alleles on estimated genomic relationships from whole genome sequence data. BMC Genet. 2015;16:24.

28. Berger S, Pérez-Rodríguez P, Veturi Y, Simianer H, los Campos G. Effectiveness of shrinkage and variable selection methods for the prediction of complex human traits using data from distantly related individuals. Ann Hum Genet. 2015;79:122-35.

29. Meuwissen THE, Luan T, Woolliams JA. The unified approach to the use of genomic and pedigree information in genomic evaluations revisited. J Anim Breed Genet. 2011;128:429-39.

30. Lee SH, Yang J, Chen GB, Ripke S, Stahl EA, Hultman CM, et al. Estimation of SNP heritability from dense genotype data. Am J Hum Genet. 2013;93:1151-5.

31. Sun X, Fernando RL, Garrick DJ, Dekkers J. Improved accuracy of genomic prediction for traits with rare QTL by fitting haplotypes. In: Proceedings of the 10th World Congress of Genetics Applied to Livestock Production; Vancouver. 2014. p. 209.

32. Lee SH, DeCandia TR, Ripke S, Yang J, the Schizophrenia Psychiatric Genome-Wide Association Study Consortium, The International Schizophrenia Consortium, et al. Estimating the proportion of variation in susceptibility to schizophrenia captured by common SNPs. Nat Genet. 2012:44:247-50.

33. Abdollahi-Arpanahi R, Pakdel A, Nejati-Javaremi A, Moradi-Shahrbabak M, Morota $\mathrm{G}$, Valente $\mathrm{BD}$, et al. Dissection of additive genetic variability for quantitative traits in chickens using SNP markers. J Anim Breed Genet. 2014;131:183-93.

34. Abdollahi-Arpanahi R, Nejati-Javaremi A, Pakdel A, Moradi-Shahrbabak M, Morota $\mathrm{G}$, Valente BD, et al. Effect of allele frequencies, effect sizes and number of markers on prediction of quantitative traits in chickens. J Anim Breed Genet. 2014;131:123-33.

35. Daetwyler HD, Villanueva B, Woolliams JA. Accuracy of predicting the genetic risk of disease using a genome-wide approach. PLoS One. 2008;3:e3395.
36. Nomura T, Honda T, Mukai F. Inbreeding and effective population size of Japanese Black cattle. J Anim Sci. 2001;79:366-70.

37. Ogawa S, Matsuda H, Taniguchi Y, Watanabe T, Nishimura S, Sugimoto Y, et al. Effects of single nucleotide polymorphism marker density on degree of genetic variance explained and genomic evaluation for carcass traits in Japanese Black beef cattle. BMC Genet. 2014;15:15.

38. Onogi A, Ogino A, Komatsu T, Shoji N, Simizu K, Kurogi K, et al. Genomic prediction in Japanese Black cattle: application of a single-step approach to beef cattle. J Anim Sci. 2014;92:1931-8.

39. Watanabe $T$, Matsuda $H$, Arakawa A, Yamada T, Iwaisaki H, Nishimura S, et al. Estimation of variance components for carcass traits in Japanese Black cattle using 50 K SNP genotype data. Anim Sci J. 2014;85:1-7

40. Haile-Mariam M, Nieuwhof GJ, Beard KT, Konstatinov KV, Hayes BJ. Comparison of heritabilities of dairy traits in Australian Holstein-Friesian cattle from genomic and pedigree data and implications for genomic evaluations. J Anim Breed Genet. 2013;130:20-31.

41. Loberg A, Dürr JW, Fikse WF, Jorjani H, Crooks L. Estimates of genetic variance and variance of predicted genetic merits using pedigree or genomic relationship matrices in six Brown Swiss cattle populations for different traits. J Anim Breed Genet. 2015. doi:10.1111/jbg.12142.

\section{Submit your next manuscript to BioMed Central and take full advantage of:}

- Convenient online submission

- Thorough peer review

- No space constraints or color figure charges

- Immediate publication on acceptance

- Inclusion in PubMed, CAS, Scopus and Google Scholar

- Research which is freely available for redistribution

Submit your manuscript at www.biomedcentral.com/submit 